\title{
Modeling Drug- and System-Related Changes in Body Temperature: Application to Clomethiazole-Induced Hypothermia, Long-Lasting Tolerance Development, and Circadian Rhythm in Rats
}

\author{
Sandra A. G. Visser, Björn Sällström, Tomas Forsberg, Lambertus A. Peletier, and \\ Johan Gabrielsson
}

PKIPD Section, DMPK \& Bioanalytical Chemistry, Local Discovery Research Area CNS \& Pain Control, AstraZeneca R\&D Södertälje, Södertälje, Sweden (S.A.G.V.); General Pharmacology, Local Discovery Research Area CNS \& Pain Control, AstraZeneca R\&D Södertälje, Sweden (T.F.); Mathematical Institute, Leiden University, Leiden, and Centrum voor Wiskunde en Informatica, Amsterdam, The Netherlands (L.A.P.); and DMPK \& Bioanalytical Chemistry, AstraZeneca R\&D Mölndal, Mölndal, Sweden (J.G.)

Received September 20, 2005; accepted December 1, 2005

\begin{abstract}
The aim of the present investigation was to develop a pharmacokinetic-pharmacodynamic model for the characterization of clomethiazole (CMZ)-induced hypothermia and the rapid development of long-lasting tolerance in rats while taking into account circadian rhythm in baseline and the influence of handling. CMZ-induced hypothermia and tolerance was measured using body temperature telemetry in male Sprague-Dawley rats, which were given s.c. bolus injections of $0,15,150,300$, and $600 \mu \mathrm{mol} \mathrm{kg}{ }^{-1}$ and $24-h$ s.c. continuous infusions of 0,20 , and $40 \mu \mathrm{mol} \mathrm{kg}{ }^{-1} \mathrm{~h}^{-1}$ using osmotic pumps. The duration of tolerance was studied by repeated injections of $300 \mu \mathrm{mol} \mathrm{kg}$ at 3- to 32-day intervals. Plasma exposure to CMZ was obtained in satellite groups of catheterized rats. Fitted population concentration-time profiles served as input for the pharmaco-
\end{abstract}

dynamic analysis. The asymmetric circadian rhythm in baseline body temperature was successfully described by a novel negative feedback model incorporating external light-dark conditions. An empirical function characterized the transient increase in temperature upon handling of the animal. A feedback model for temperature regulation and tolerance development allowed estimation of $\mathrm{CMZ}$ potency at $30 \pm 1 \mu \mathrm{M}$. The delay in onset of tolerance was estimated via a series of four transit compartments at $7.6 \pm 2 \mathrm{~h}$. The long-lasting tolerance was assumed to be caused by inactivation of a mediator with an estimated turnover time of $46 \pm 3$ days. This multicomponent turnover model was able to quantify the CMZ-induced hypothermia, circadian rhythm in baseline, and rapid onset of a long-lasting tolerance to $\mathrm{CMZ}$ in rats.
Clomethiazole (CMZ) has sedative, hypnotic, anticonvulsive, and neuroprotective properties and has been used clinically in elderly patients as a useful sedative/hypnotic for 40 years (Green, 1998). In a recent investigation, we showed that CMZ is able to induce hypothermia in rats and that even a single injection of CMZ rapidly induces complete tolerance lasting for more than 10 days, with a $50 \%$ return of effect

Preliminary results were presented at the annual meeting of the Population Approach Group Europe (PAGE), June 17-18, 2004, Uppsala, Sweden [Visser SAG, Sällström B, Forsberg T, Peletier LA, and Gabrielsson J (2004) Modeling of drug- and system-related changes in body temperature: application to drug-induced hypothermia, long-lasting tolerance development and circadian rhythm in body temperature [PAGE 13 (2004) Abstract 512. www.pagemeeting.org/?abstract $=512]$.

Article, publication date, and citation information can be found at http://jpet.aspetjournals.org.

doi:10.1124/jpet.105.095224. after a month (Visser et al., 2005). This observation of tolerance partly explained why conflicting reports exist regarding the ability of CMZ to induce hypothermia and the relationship between hypothermia and neuroprotective properties in vivo (Cross et al., 1991; Green, 1998; Chaulk et al., 2003). For CMZ, it was observed that the tolerance is induced directly by the drug, rather than via a decrease in body temperature, because hypothermic tolerance to CMZ did not affect hypothermic responses to other drugs and could not be attenuated (Visser et al., 2005). Moreover, the tolerance appeared to last for more than 15 days, which suggests that CMZ downregulated a necessary but so far unknown mediator, which by itself has a slow turnover. In the present investigation the objective was to quantify the CMZ-induced hypothermia and the rapid onset of long-lasting tolerance by developing a pharmacokinetic-pharmacodynamic (PK/PD) model. 
The regulation of body temperature is a complex homeostatic control mechanism, the purpose of which is to maintain body temperature at a constant level (Simon et al., 1986). It is affected by, among other things, the neurotransmitter systems of serotonin, GABA, glutamate, and dopamine and modulated by inflammatory processes (Zarrindast and Oveissi, 1988; Corbett et al., 1990; Briese, 1998; Salmi and Ahlenius, 1998; Blatteis, 2000; Perachon et al., 2000). The effect of a drug on body temperature is the result of multiple time-dependent processes with their own specific rate constants, such as compound distribution and elimination, temperature baseline behavior, the effects on body temperature regulation, and the development of tolerance. The pharmacokinetics can practically be determined and quantified separately. Information on drug exposure can then be used as the driving force of the pharmacodynamics. For the modeling of body temperature modulation, a turnover model or a model for homeostatic regulation of body temperature has been applied, accounting for the time delay between maximum exposure and effect (Ackerman et al., 1964; Gabrielsson and Weiner, 1997; Zuideveld et al., 2001). For the CMZ-induced effects on body temperature and tolerance development, a systematic approach based on previously described models was adopted to build a pharmacodynamic model composed of various subsystems with their specific rate constants.

It is also commonly known that body temperature is subject to circadian rhythm at around a constant level with a $1^{\circ} \mathrm{C}$ lower temperature during the day compared with the night in rats (Lobo et al., 1999; Benstaali et al., 2001). So far, models based on trigonometric functions such as the cosine function or Fourier series have been applied for the description of circadian rhythm (Hempel et al., 1998; Chakraborty et al., 1999). However, here we apply a novel feedback model for circadian rhythm, which is described in more detail elsewhere (Sällström et al., 2005). This is a generic model, which is able to describe the (asymmetric) biorhythm of biomarkers.

Tolerance development can be considered as a homeostatic process of the body to reduce the effect of altered conditions, such as receptor down-regulation and adaptation of signaltransduction processes, during drug administration. The best studied is the glucose-insulin system (Ackerman et al., 1964), but nicotine and nitroglycerin tolerance have also been well studied (Porchet et al., 1988; Bauer et al., 1997; Fattinger et al., 1997). A number of tolerance models have been proposed and applied, depending on the proposed mechanism of action, such as the formation of antagonistic metabolites, tolerance compartment, counter-regulation, feedback, and pool and precursor models (Holford and Sheiner, 1982; Ekblad and Licko, 1984; Mandema and Wada, 1995; Movin-Osswald and Hammarlund-Udenaes, 1995; Wakelkamp et al., 1996; Gabrielsson and Weiner, 1997; Gardmark et al., 1999).

In the present investigation, the CMZ-induced hypothermia and the rapid onset of long-lasting tolerance was quantified by development of a PK/PD model consisting of different submodels for circadian rhythm in baseline, handling events, drug effects, and tolerance development.

\section{Materials and Methods}

Temperature and Exposure Measurements. The in vivo experiments have been described previously (Visser et al., 2005). In short, male Sprague-Dawley rats $[n=117$, weight (mean \pm S.D.):
$301 \pm 23$ g; B\&K Universal AB, Sollentuna, Sweden)] were used in pharmacodynamic experiments 7 days after implantation of a telemetric transmitter into the peritoneal cavity (TA10TA-F20; Data Sciences, St. Paul, MN). Satellite groups of male animals were used in separate pharmacokinetic experiments 3 days after implantation of a catheter in the carotid artery and the telemetric transmitter in the peritoneal cavity. The animals were kept in rooms where the artificial light followed a 12-h cycle (lights on at 6:00 AM). Throughout the experiments the rats had access to food and tap water ad libitum. Ethical permission was obtained from the Stockholm Animal Ethics Committee, Sweden. Body temperature was recorded 4 days before dosing until 4 to 6 days after the end of dosing. The telemetry signals were measured every 2 min for a 10 -s period and signaled to a receiver. Using the software package Dataquest ART 2.2 (Data Sciences), the temperature data were processed, analyzed, and visualized. The animals were disturbed as little as possible throughout the experiment by allowing entry to the experimental room only during dosing and care. For the assessment of CMZ exposure, blood samples were taken at predefined intervals, with up to $2 \mathrm{ml}$ of blood being withdrawn. The plasma samples were analyzed using high-performance liquid chromatography-UV. Precision and accuracy were previously reported (Visser et al., 2005).

Drugs, Dosages, and Dosing. Clomethiazole (clomethiazole edisilate, mol. wt. $523.46 \mathrm{~g} \mathrm{~mol}^{-1}$, the denoted test compound) was obtained from Compound Management (AstraZeneca R\&D Södertälje, Södertälje, Sweden). The concentration of CMZ in all solutions was calculated on the basis of the clomethiazole base (mol. wt. 161.7 $\left.\mathrm{g} \mathrm{mol}^{-1}\right)$. All injection solutions were made in saline $\left(9 \mathrm{mg} \mathrm{ml}^{-1}\right.$, Braun Medical AB, Bromma, Sweden) on the day of the experiment. The rats were randomly assigned to dosing groups and received a weight-adjusted s.c. injection dose or saline as vehicle treatment. The dosing design for the characterization of the hypothermia and tolerance development is given in Table 1 . A validation data set was obtained in an additional experiment, in which CMZ was administered three times at an interval of 15 days. Alzet osmotic pumps (model 2001D; Scanbur BK AB, Sollentuna, Sweden) with an average pump rate of $7.4 \mu \mathrm{l} \mathrm{h}^{-1}$ were used for s.c. drug infusion at a continuous rate. The osmotic pumps were filled with a solution of CMZ in saline. The pumps were preincubated in saline for a minimum of $2.5 \mathrm{~h}$ at $37^{\circ} \mathrm{C}$ before being inserted s.c. into the neck of the rat under light isoflurane anesthesia.

Data Analysis. A PK/PD model was developed to describe body temperature in vehicle- and CMZ-treated rats (Fig. 1). It consists of a pharmacokinetic model describing the exposure profile after drug administration, a circadian rhythm (biorhythm) model describing the asymmetric day/night temperature profile with a correction for stress-induced temperature elevation seen when animals were handled for drug administration or surgery, and a turnover model comprising CMZ-induced hypothermia and tolerance development.

Exposure to CMZ. The time course of the CMZ plasma concentration after i.v. infusion has been described previously by a twocompartment disposition model with capacity-limited elimination (Gabrielsson and Weiner, 1997). These data were included to allow better estimation of the maximum metabolic rate and the MichaelisMenten constant after s.c. dosing. The bolus injection was administered to a s.c. compartment at time 0 , and the decrease in the amount of CMZ at the s.c. compartment was described by:

$$
\frac{d A_{\mathrm{sc}}}{d t}=-K_{\mathrm{a}} \cdot A_{\mathrm{sc}}
$$

where $K_{\mathrm{a}}$ is the absorption rate constant. The amount of CMZ in the s.c. compartment during the 24-h s.c. continuous infusion via an osmotic pump was described by:

$$
\frac{d A_{\mathrm{sc}}}{d t}=\frac{D_{\mathrm{sc}}}{t_{\mathrm{inf}}}-K_{\mathrm{a}} \cdot A_{\mathrm{sc}}
$$


TABLE 1

Dosing groups for characterization and validation of the hypothermia and the onset and duration of tolerance to CMZ

Solutions of $1.5 \mathrm{ml} / \mathrm{kg}$ with various concentrations of CMZ were injected s.c. The concentrations of CMZ in the osmotic pump were 680 and $1350 \mu$ mol ml ${ }^{-1}$, respectively. Groups denoted $X \mathrm{~A}$ received treatment only on day 1 , whereas groups $X \mathrm{~B}$ received treatment on both days 1 and 3.

\begin{tabular}{|c|c|c|c|c|c|c|c|}
\hline Group & Day & Treatment $t=0$ & Route & Day & Treatment & Route & $n$ \\
\hline & & $\mu \mathrm{mol} \mathrm{kg^{-1 }}$ & & & $\mu \mathrm{mol} \mathrm{kg}{ }^{-1}$ & & \\
\hline \multicolumn{8}{|c|}{ CMZ-mediated hypothermia and onset of tolerance } \\
\hline $1 \mathrm{~A} / 1 \mathrm{~B}$ & 1 & Vehicle & s.c. & 3 & 300 & s.c. & $6 / 9$ \\
\hline 2 & 1 & 15 & s.c. & & & & 6 \\
\hline 3 & 1 & 150 & s.c. & & & & 6 \\
\hline 4 & 1 & 300 & s.c. & 3 & 300 & s.c. & 6 \\
\hline 5 & 1 & 600 & s.c. & & & & 6 \\
\hline $6 \mathrm{~A} / 6 \mathrm{~B}$ & 1 & Vehicle & Pump & 3 & 300 & s.c. & $6 / 3$ \\
\hline $7 \mathrm{~A} / 7 \mathrm{~B}$ & 1 & 20 & Pump & 3 & 300 & s.c & $5 / 6$ \\
\hline $8 \mathrm{~A} / 8 \mathrm{~B}$ & 1 & 40 & Pump & 3 & 300 & s.c. & $5 / 6$ \\
\hline \multicolumn{8}{|c|}{ Onset and duration of tolerance to CMZ } \\
\hline 9 & 1 & 300 & s.c. & 3 & 300 & s.c. & 6 \\
\hline 10 & 1 & 300 & s.c. & 6 & 300 & s.c. & 6 \\
\hline 11 & 1 & 300 & s.c. & 10 & 300 & s.c. & 6 \\
\hline 12 & 1 & 300 & s.c. & 16 & 300 & s.c. & 6 \\
\hline 13 & 1 & 300 & s.c. & 24 & 300 & s.c. & 6 \\
\hline 14 & 1 & 300 & s.c. & 32 & 300 & s.c. & 6 \\
\hline \multicolumn{8}{|l|}{ Validation data set } \\
\hline 15 & 1 & 300 & s.c. & $15+32$ & 300 & s.c. & 5 \\
\hline
\end{tabular}

\section{A: CMZ pharmacokinetics}

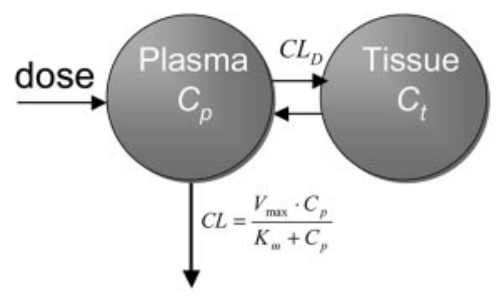

\section{B: Diurnal variation and handling}

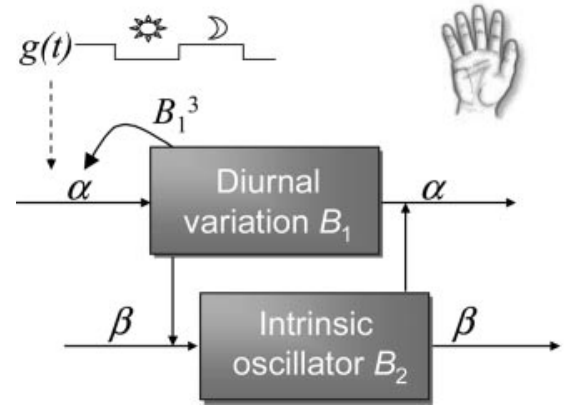

\section{C: Temperature regulation and} tolerance development

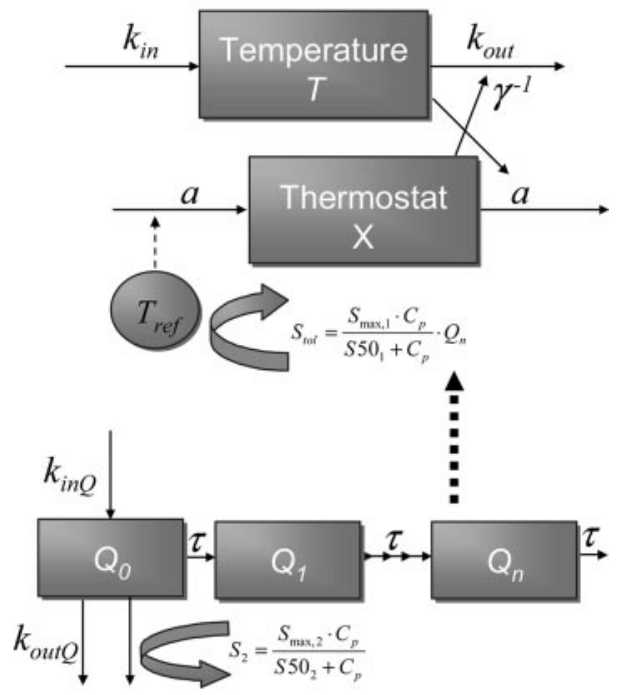

Fig. 1. The proposed pharmacokinetic-pharmacodynamic model consisting of three components. A, CMZ pharmacokinetics with a capacity-limited clearance. B, circadian rhythm in baseline and handling-induced elevation of temperature (Sällström et al., 2005). C, temperature regulation according to the set-point model (Zuideveld et al., 2001) including the tolerance model with a slow turnover and a delay in onset via transit compartments. The data were analyzed in a sequential way. First, the pharmacokinetics was analyzed, and the population exposure profiles were simulated at each time point of the temperature observations (A). Second, the fitted population vehicle response including circadian rhythm was used for rescaling the body temperature after CMZ treatment (B). Last, the body temperature and tolerance development after drug administration was modeled (C). where $D_{\text {sc }}$ equals the total amount of CMZ infused under the duration of implantation $\left(t_{\text {inf }}\right)$ of the osmotic pump. After removal of a pump, the amount in the s.c. compartment equals zero. The CMZ concentrations in the central $(p)$ and peripheral $(t)$ compartments were described by:

$$
\left\{\begin{array}{l}
\frac{d C_{\mathrm{p}}}{d t}=\left(F \cdot K_{\mathrm{a}} \cdot A_{\mathrm{sc}}-\mathrm{CL}_{\mathrm{D}} \cdot C_{\mathrm{p}}+\mathrm{CL}_{\mathrm{D}} \cdot C_{\mathrm{t}}-\mathrm{CL}\right) / V_{\mathrm{c}} \\
\frac{d C_{\mathrm{t}}}{d t}=\left(\mathrm{CL}_{\mathrm{D}} \cdot C_{\mathrm{p}}-\mathrm{CL}_{\mathrm{D}} \cdot C_{\mathrm{t}}\right) / V_{\mathrm{t}}
\end{array}\right.
$$

where $F$ is the s.c. bioavailability, $\mathrm{CL}_{\mathrm{D}}$ is the intercompartmental distribution term, and $V_{\mathrm{c}}$ and $V_{\mathrm{t}}$ are the volumes of the central and peripheral compartments, respectively. The capacity-limited clearance (CL) is defined as:

$$
\mathrm{CL}=\frac{V_{\max } \cdot C_{\mathrm{p}}}{K_{\mathrm{m}}+C_{\mathrm{p}}}
$$

where the parameters $V_{\max }$ and $K_{\mathrm{m}}$ define the maximum metabolic rate and the Michaelis-Menten constant, respectively.

Baseline Behavior. A turnover model was developed for the description of asymmetric circadian rhythm in baseline temperature in rats (Sällström et al., 2005). The biorhythm model consists of two interconnected compartments $B_{1}$ and $B_{2}$. The input and output rates of the compartments are governed by:

$$
\left\{\begin{array}{l}
\frac{d B_{1}}{d t}=\alpha \cdot\left(B_{1}-B_{2}\right)-B_{1}^{3}+g(t) \\
\frac{d B_{2}}{d t}=\beta \cdot\left(B_{1}-B_{2}\right)
\end{array}\right.
$$


where $\alpha$ and $\beta$ are first-order rate constants and the timekeeper $g(t)$ describes the external light conditions following a squared wave function:

$$
g(t)=\left\{\begin{array}{lll}
0 & 6 \text { AM-6 PM } & \text { (light-on) } \\
d & 6 \text { PM-6 AM } & \text { (light-off) }
\end{array}\right.
$$

where $d$ represents the intensity of a night stimulus because the animals are more active at night. The model can account for a low but slowly increasing temperature during the day and a high and relatively stable temperature during the night. The baseline body temperature was defined as:

$$
T_{\text {baseline }}=T_{\text {ref }} \cdot\left(1+\mathrm{amp} \cdot B_{1}\right)
$$

where $T_{\text {ref }}$ is the reference temperature level and amp adjusts the amplitude of the circadian rhythm in $B_{1}$ around the reference temperature. Hence, $T_{\text {baseline }}$ represents the model for circadian rhythm, describing the day/night temperature profile of normal rats in an artificial 12/12-h light/dark cycle environment.

Vehicle Treatment. It was observed that handling of the animals caused a temporary temperature increase that was independent of dose level. Therefore, for the individual estimation of the temperature profile upon vehicle treatment (s.c. injection or continuous pump), the model was corrected by an empirical function $H D$ mimicking this handling effect:

$$
H D=k_{\mathrm{HD}} \cdot\left(t-t_{\mathrm{HD}}\right) \cdot P \cdot e^{-k_{\mathrm{HD}} \cdot\left(t-t_{\mathrm{HD}}\right)} \quad \text { when } t>t_{\mathrm{HD}}
$$

where $P$ determines the magnitude of the temperature elevation, whereas $k_{\mathrm{HD}}$ determines the rate of the appearance and disappearance of the temporary temperature elevation and $t_{\mathrm{HD}}$ is set to the time of handling. Hence, the temperature profile during vehicle treatment is defined as:

$$
T_{\text {vehicle }}=T_{\text {ref }} \cdot H D \cdot\left(1+\mathrm{amp} \cdot B_{1}\right) .
$$

Drug-Induced Hypothermia. The model of temperature regulation applied in this investigation has been described previously and uses concepts of turnover principles and feedback regulation (Ackerman et al., 1964; Gabrielsson and Weiner, 1997; Zuideveld et al., 2001, 2004). It is based on a turnover model for body temperature (T) combined with a thermostat-like regulation of body temperature $(X)$ :

$$
\left\{\begin{array}{l}
\frac{d T}{d t}=k_{\text {in }}-k_{\text {out }} \cdot T \cdot X^{-\gamma} \\
\frac{d X}{d t}=a \cdot\left(T_{\text {drug }}-T\right)
\end{array}\right.
$$

The parameters $k_{\text {in }}$ and $k_{\text {out }}$ are the turnover rate and the fractional turnover rate governing the production and loss of heat, respectively. The parameter $T_{\text {drug }}$ is dependent on a function of the drug plasma concentration and is defined by:

$$
T_{\text {drug }}=T_{\text {ref }} \cdot(1-f(C))
$$

where $T_{\text {ref }}$ is the reference temperature level in the absence of the drug and baseline variation (see eq. 6 ). The relationship between the plasma concentration and the stimulus $\left(S_{1}\right)$ on the temperature system is defined by:

$$
f(C)=S_{1}=\frac{S_{\max , 1} \cdot C_{\mathrm{p}}}{S C_{50,1}+C_{\mathrm{p}}}
$$

where $C_{\mathrm{p}}$ is the plasma concentration of CMZ, $S C_{50,1}$ is the concentration producing half the maximum stimulus and $S_{\max , 1}$ is the maximum stimulus. Because CMZ induced a temperature decrease down to $31^{\circ} \mathrm{C}$ at the highest dose $\left(600 \mu \mathrm{mol} \mathrm{kg}{ }^{-1}\right)$, it was assumed that CMZ can exert the maximum possible reversible effect on temperature, so $S_{\max , 1}$ was fixed at 1 .

The model for temperature regulation with four system parame- ters to be estimated can lead to parameter unidentifiability (for details, see Zuideveld et al., 2001). In a rescaling strategy, one parameter can be eliminated by making the system dimensionless. The dimensionless system $(x, y)$ is obtained by:

$$
x=\frac{X}{X_{0}} \quad \text { and } \quad y=\frac{T}{T_{0}}
$$

where $T_{0}$ is equal to $T_{\text {ref }}$ and $X_{0}$ is the reference signal of $X$ :

$$
X_{0}=\left(\frac{k_{\text {out }} \cdot T_{\text {ref }}}{k_{\text {in }}}\right)^{1 / \gamma} .
$$

The dimensionless system is given by:

$$
\left\{\begin{array}{l}
\frac{d y}{d t}=B\left(1-y \cdot x^{-\gamma}\right) \\
\frac{d x}{d t}=A \cdot\left(\left(1-S_{1}\right)-y\right)
\end{array}\right.
$$

where the new parameters $A$ and $B$ are defined by:

$$
A=\frac{a \cdot T_{0}}{X_{0}}=a \cdot\left(\frac{k_{\text {in }}}{k_{\text {out }}}\right)^{1 / \gamma} \cdot T_{0}^{1-(1 / \gamma)}
$$

and

$$
B=\frac{k_{\text {in }}}{T_{\text {ref }}}
$$

The temperature model in eq. 14 can describe drug-induced hypothermia. It must be noted that eq. 14 is only valid in the absence of tolerance development. In the next section, eq. 14 will be elaborated on further with a model for tolerance development.

Tolerance Model. It was proposed that CMZ induces long-lasting tolerance by the irreversible removal of an unknown mediator $Q_{0}$ (e.g., irreversible degradation of a receptor or second messenger), which has a slow turnover (Visser et al., 2005). Preliminary analysis of the data showed also that the tolerance development did not occur instantaneously but needed time to develop. To estimate this delay, it was assumed that $Q_{0}$ was cascaded through a number of transit compartments before it reduced the effect of CMZ on the temperature (Sun and Jusko, 1998). The proposed full model for tolerance is given by:

$$
\left\{\begin{aligned}
\frac{d Q_{0}}{d t} & =k_{\text {in } Q}-k_{\text {out } Q} \cdot Q_{0}-S_{2} \cdot Q_{0} \\
\frac{d Q_{1}}{d t} & =\frac{1}{\tau}\left(Q_{0}-Q_{1}\right) \\
\frac{d Q_{2}}{d t} & =\frac{1}{\tau}\left(Q_{1}-Q_{2}\right) \\
\cdots & \frac{d Q_{\mathrm{n}}}{d t}=\frac{1}{\tau}\left(Q_{(\mathrm{n}-1)}-Q_{\mathrm{n}}\right)
\end{aligned}\right.
$$

The parameters $k_{\text {in } Q}$ and $k_{\text {out } Q}$ are the turnover rate and the fractional turnover rate governing the production and loss of $Q_{0}$, respectively. $\tau$ reflects the transit time of $Q_{0}$ to $Q_{1}$ and from $Q_{1}$ to $Q_{2}$, etc. $S_{2}$ is a sigmoidal function of the plasma concentration:

$$
S_{2}=\frac{S_{\max , 2} \cdot C_{\mathrm{p}}}{S C_{50,2}+C_{\mathrm{p}}}
$$

in which $S_{\max , 2}$ is the maximum rate of loss from compartment $Q_{0}$ and $S C_{50,2}$ is the concentration giving half the maximum stimulus. $Q_{0}$ is normalized to unity (=1) which means that $k_{\text {in } Q}=k_{\text {out } Q}$. The turnover time of $Q_{0}$ and the half-life of the irreversible emptying of $Q_{0}$ are defined by, respectively:

$$
t 1 / 2 k_{\text {out } Q}=\frac{\ln 2}{k_{\text {out } Q}}
$$


and

$$
t_{1 / 2} S_{\max , 2}=\frac{\ln 2}{S_{\max , 2}}
$$

The complete model for temperature regulation and acute tolerance development after administration of CMZ is defined by:

$$
\left\{\begin{array}{l}
\frac{d y}{d t}=B\left(1-y \cdot x^{-\gamma}\right) \\
\frac{d x}{d t}=A \cdot\left(\left(1-S_{\mathrm{tol}}\right)-y\right)
\end{array}\right.
$$

in which $S_{\text {tol }}$ is given by:

$$
S_{\mathrm{tol}}=S_{1} \cdot Q_{\mathrm{n}}
$$

In this way, tolerance development occurs with a delay and the total stimulus $\left(S_{\text {tol }}\right)$ is equal to 0 when CMZ exposure is 0 , and therefore tolerance will not affect the body temperature itself but only by means of CMZ.

Modeling Procedure. A sequential modeling approach was adapted. All fitting procedures were performed using NONMEM V. First, the individual concentration-time profiles were fitted simultaneously to eq. 2. Population pharmacokinetic parameter estimates were used to simulate concentrations to the temperature measurements. For the determination of the circadian rhythm in temperature, 6 days of baseline recordings (each $2 \mathrm{~min}$ ) in vehicle groups (1A and $6 \mathrm{~A}, n=6$ per group) were averaged to a 24 -h profile and fitted to eq. $6 ; 3 \mathrm{~h}$ of data after the time of handling were removed to avoid interference.

In the subsequent analysis, all temperature data were reduced to one observation per $20 \mathrm{~min}$ to reduce computational time. Individual temperature profiles in all vehicle groups were analyzed by means of eq. 8, allowing a different magnitude of temperature elevation after s.c. injection and implantation of the pump. The population parameter estimates for the circadian rhythm and handling were used to simulate the vehicle baseline temperature $\left(T_{\text {vehicle }}\right)$. This profile was used to normalize the temperature during drug treatment to vehicle treatment. The temperature data were rescaled following:

$$
T_{\text {rescaled }}=\frac{T_{\text {obs }}-T_{\min }}{T_{\text {vehicle }}-T_{\min }} .
$$

The rescaling of the temperature data to make the data dimensionless was necessary to enable the use of the reduced parameter temperature model (see eq. 21). For graphical purposes, the final temperature predictions (pred) were recalculated to $T_{\text {body,pred }}$ via:

$$
T_{\mathrm{body}, \operatorname{pred}}=\operatorname{pred} \cdot T_{\text {vehicle }}-\operatorname{pred} \cdot T_{\min }+T_{\min } .
$$

The interindividual variability of all estimated parameters was modeled by an exponential equation:

$$
P_{\mathrm{i}}=\theta_{1} \cdot \exp \left(\eta_{\mathrm{i}}\right)
$$

where $\theta$ is the population estimate for parameter $P, P_{\mathrm{i}}$ is the individual estimate, and $\theta_{\mathrm{i}}$ is the random deviation of $P_{\mathrm{i}}$ from $P$. The values of $\eta_{\mathrm{i}}$ are assumed to be independently normally distributed with mean zero and variance $\omega^{2}$. The covariance structure of the variability parameters was assumed to be diagonal. The residual error in the plasma drug concentration and pharmacodynamics was characterized by a constant coefficient of variation error model:

$$
y_{\mathrm{m}_{\mathrm{ij}}}=y_{\mathrm{p}_{\mathrm{ij}}} \cdot\left(1+\varepsilon_{\mathrm{ij}}\right)
$$

where $y_{p_{i j}}$ represents the $j$ th observation for the $i$ th individual predicted by the model. $y_{m_{i j}}$ represents the predicted concentration or temperature, and $\epsilon_{\mathrm{ij}}$ accounts for the residual deviation of the modelpredicted value from the observation. The values for $\epsilon$ were assumed to be independently normally distributed with mean zero and vari- ance $\sigma^{2}$. The first-order estimation method (FOCE interaction) was used to estimate the population $\theta, \omega^{2}$, and $\sigma^{2}$ for the pharmacokinetic analysis. A centering first-order conditional estimation method was used for the pharmacodynamic analysis. Individual parameter estimates were obtained in a Bayesian post hoc step.

\section{Results}

Pharmacokinetics. Concentration-time profiles of CMZ displayed typical nonlinear kinetics with increasing doses of the test compound. A two-compartment model with capacitylimited elimination described the exposure profiles best. The population and individual fits are shown in Fig. 2. Population parameter estimates are summarized in Table 2. Intravenous infusion data (Gabrielsson and Weiner, 1997) were used to estimate the Michaelis-Menten constants. The bioavailability was around $100 \%$. The absorption rate from the s.c. site $\left(K_{\mathrm{a}}\right)$ decreased with increases in the CMZ dose (Table 2). The CMZ concentration in the injection solutions for 15, 150, 300 , and $600 \mu \mathrm{mol} \mathrm{kg}{ }^{-1}$ s.c. bolus doses and 20 and $40 \mu \mathrm{mol}$ $\mathrm{kg}^{-1} \mathrm{~h}^{-1}$ s.c. infusion doses were $10,100,200,400,680$, and $1380 \mu \mathrm{mol} \mathrm{ml}{ }^{-1}$, respectively. The $\mathrm{pH}$ decreased in this concentration range from 7 to 1 and was not adjusted for reasons of low solubility at higher $\mathrm{pH}$.

Baseline Temperature. Six days of temperature observations in groups $1 \mathrm{~A}$ and $6 \mathrm{~A}$ were averaged for all individuals ( $n=6 \times 6$ days $=36$ days per treatment group). Three hours of observations were removed after each handling event to avoid influence of handling. Baseline profiles were fitted using eq. 6 . The asymmetric temperature baseline is shown in Fig. 3A. During the day a gradual increase in temperature was observed, followed by an abrupt increase in temperature when the lights were turned off. During the night, the body temperature was reasonably stable around $38^{\circ} \mathrm{C}$ and de-
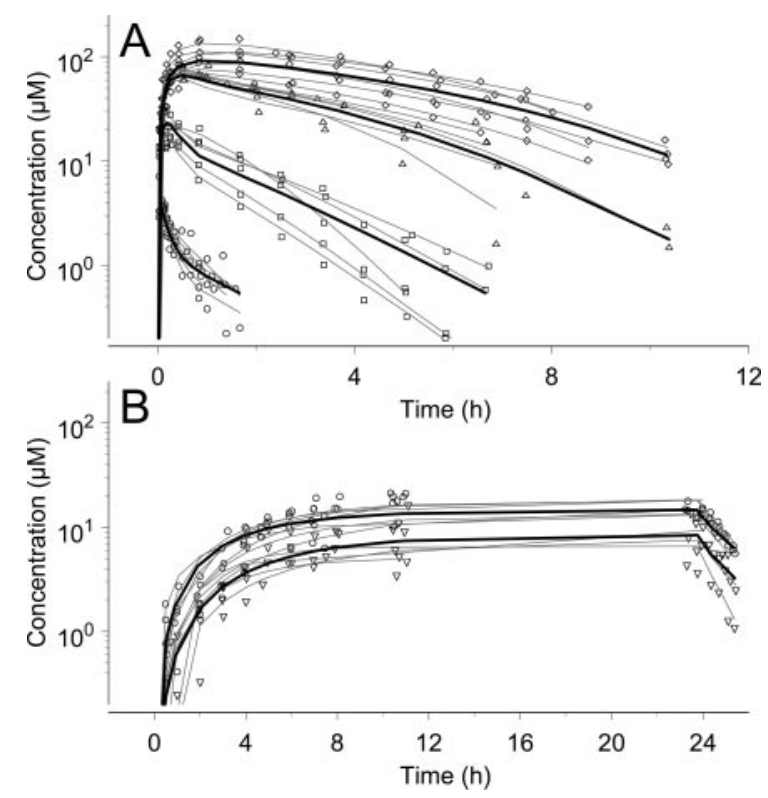

Fig. 2. Individual observed (markers) and fitted individual (thin lines) and population (thick lines) $\mathrm{CMZ}$ plasma concentrations after s.c. injec-

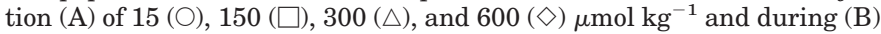
24-h osmotic pump delivery of $20(\nabla)$ and $40(\bigcirc) \mu \mathrm{mol} \mathrm{kg}^{-1} \mathrm{~h}^{-1}$. Osmotic pumps were implanted between 0 and $24 \mathrm{~h}$. Note the different time scale. The steady-state plasma concentrations of CMZ were 8 and $16 \mu \mathrm{M}$ for the 20 and $40 \mu \mathrm{mol} \mathrm{h}-1 \mathrm{~kg}^{-1}$ infusion rates, respectively. 
TABLE 2

Population pharmacokinetic parameter estimates for capacity-limited elimination

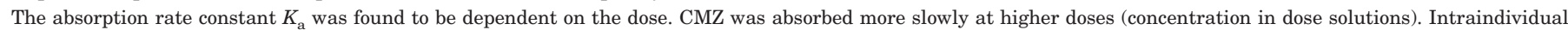
variability was $20 \%$.

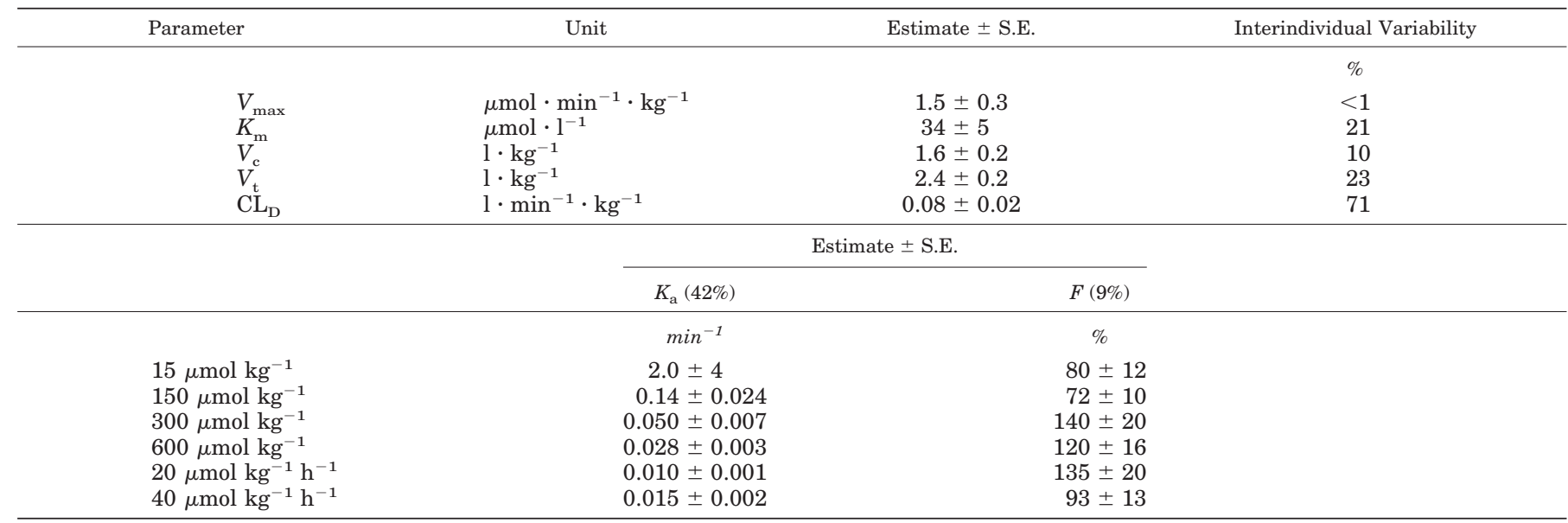
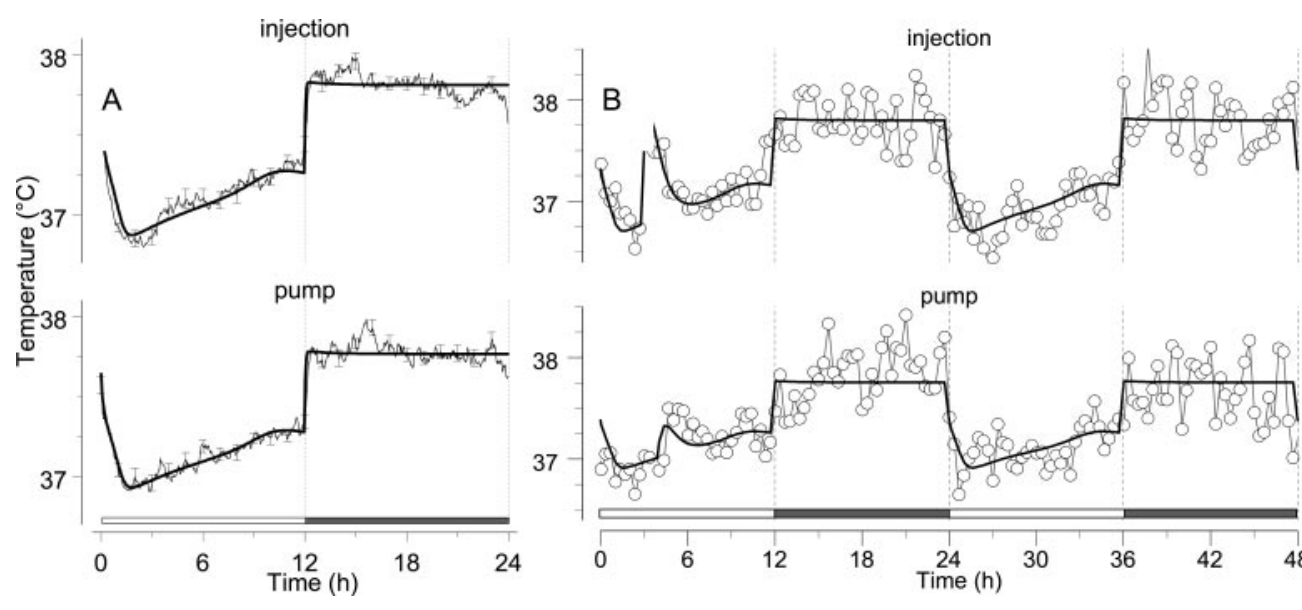

TABLE 3

Population parameter estimates for the description of the circadian rhythm in body temperature baseline and the temperature profile after vehicle treatment

Intraindividual variability was $<1 \%$.

\begin{tabular}{lccc}
\hline Parameter & Unit & Estimate \pm S.E. & $\begin{array}{c}\text { Interindividual } \\
\text { Variability (\%) }\end{array}$ \\
\hline$\alpha$ & $\mathrm{h}^{-1}$ & $0.90 \pm 0.06$ & \\
$\beta$ & $\mathrm{h}^{-1}$ & $0.66 \pm 0.06$ & \\
$T_{\text {ref }}$ & ${ }^{\circ} \mathrm{C}$ & $37.2 \pm 0.02$ & $<1$ \\
$\operatorname{amp}$ & & $0.04 \pm 0.01$ & 11 \\
$D$ & & $0.06 \pm 0.01$ & \\
$k_{\mathrm{HD}}$ & $\mathrm{h}^{-1}$ & $1.8 \pm 0.6$ & 146 \\
$\mathrm{P}$ (pump) & & $0.004 \pm 0.004$ & 62 \\
$\mathrm{P}$ (injection) & & $0.03 \pm 0.01$ & \\
\hline
\end{tabular}

creased in 1 to $2 \mathrm{~h}$ to $37^{\circ} \mathrm{C}$ when the lights were turned on. Population parameter estimates can be found in Table 3.

Vehicle Treatment. Two days of temperature recordings in the vehicle treatment groups $(1 \mathrm{~A}, 1 \mathrm{~B}, 6 \mathrm{~A}$, and $6 \mathrm{~B})$ were analyzed simultaneously using eq. 8, allowing a different magnitude of temperature elevation after s.c. injection $(n=$ 15 ) and implantation of the pump $(n=9)$. Injection or implantation of the pump occurred between 9:00 and 12:00 AM on day 1 . The population parameter estimates are given in Table 3. Two typical profiles are shown for s.c. vehicle injection and implantation of the pump in Fig. 3B. The temporary
Fig. 3. Left, averaged (mean \pm S.E.) and fitted profiles for the baseline profile. The average profiles were derived by averaging 6 consecutive days of six animals ( $n=36$ days) in each vehicle group (1A and $6 \mathrm{~A}$ ). Three hours of data were removed after handling events. Right, two typical temperature profiles after s.c. injection of vehicle and implantation of an osmotic pump loaded with vehicle. Markers represent observations and lines for the fitted baseline following eq. 7. The white/black bar represents the light/ dark cycle, and time $=0$ represents 6:00 AM. Drug administration for these individuals occurred between 10:10 AM and 10:30 AM, respectively. elevation of temperature after administration varies between animals and is three times higher after s.c. injection than after implantation of the pump.

Drug-Induced Hypothermia and Tolerance. The population prediction for the vehicle response was used to rescale the temperature data after drug treatment (see eq. 23). The exposure-related decrease in body temperature after s.c. bolus injections of 15, 150, 300, and $600 \mu \mathrm{mol} \mathrm{kg}{ }^{-1} \mathrm{CMZ}$ or following a 24 -h s.c. continuous infusion of 0,20 , or 40 $\mu \mathrm{mol} \mathrm{kg} \mathrm{kg}^{-1} \mathrm{~h}^{-1} \mathrm{CMZ}$ could simultaneously be described by the temperature-tolerance model (eq. 21). The observed and fitted time-effect profiles are shown in Fig. 4 and all population pharmacodynamic parameter estimates are given in Table 4 .

The differences in profiles after a s.c. bolus injection or s.c. continuous infusion were crucial for determination of the number of transit compartments. For the s.c. bolus injections, a clockwise hysteresis was observed and the return phase shifted to the right with increasing doses, indicating the tolerance development. In contrast, for the s.c. continuous infusion, a counterclockwise hysteresis was observed (Fig. 5). This difference arose because of the pharmacokinetics being the rate-limiting step for the s.c. continuous infusions, whereas for the s.c. bolus injections, the time constants in temperature regulation were rate-limiting. A delay for 


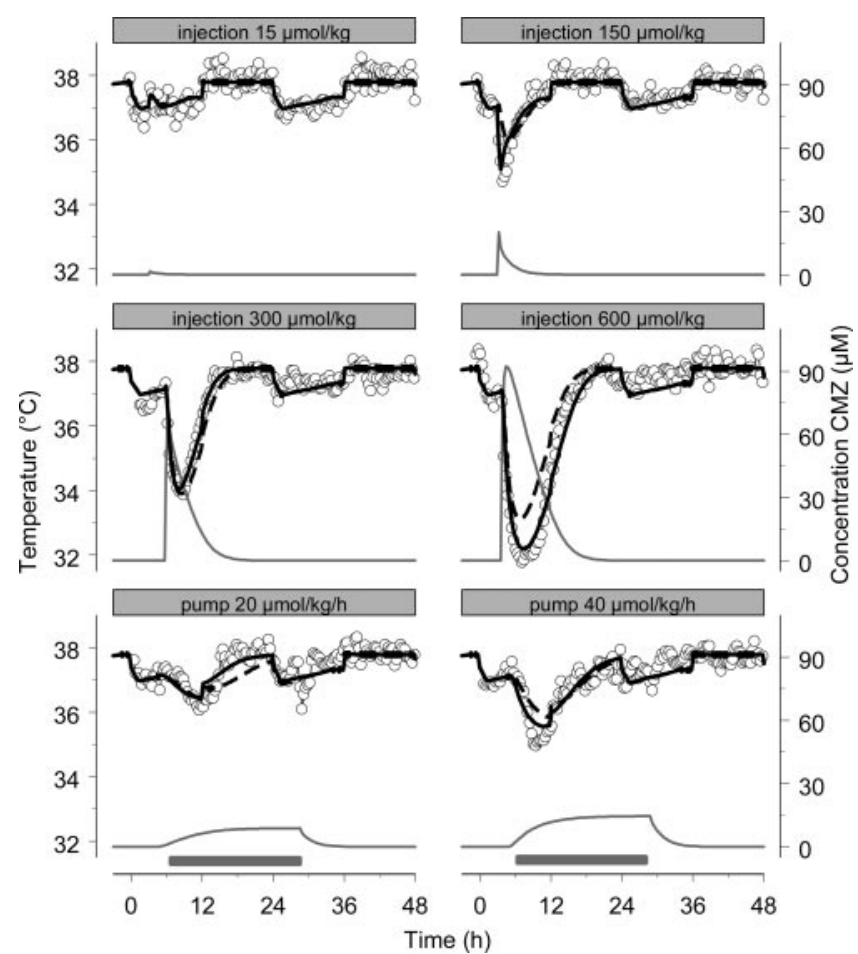

Fig. 4. Individual observed (markers) and fitted individual (solid lines) and population (dotted lines) temperature profiles for typical individuals after a single s.c. injection and implantation of an osmotic pump. The predicted CMZ concentration is shown as a gray line scaled to the right $y$-axis. Horizontal gray bars represent implantation of osmotic pump. $T=$ 0 represents 6:00 AM. Drug administration occurred between 9:00 and 12:00 AM.

TABLE 4

Population parameter estimates for the estimation drug- and systemrelated parameters for temperature regulation and tolerance Intraindividual variability was $6 \%$

\begin{tabular}{llcc}
\hline \multicolumn{1}{c}{ Parameter } & \multicolumn{1}{c}{ Unit } & Estimate \pm S.E. & $\begin{array}{r}\text { Interindividual } \\
\text { Variability (\%) }\end{array}$ \\
\hline$S_{\max , 1}$ & & 1 (fixed) & \\
$S C_{50,1}$ & $\mu \mathrm{M}$ & $30 \pm 1$ & 48 \\
$S_{\text {max }, 2}$ & $\mathrm{~h}^{-1}$ & $5.2 \pm 1.7$ & \\
$S C_{50,2}$ & $\mu \mathrm{M}$ & $69 \pm 57$ & \\
$k_{\text {in }}$ & ${ }^{\circ} \mathrm{C} \cdot \min ^{-1}$ & $2.7 \pm 0.9$ & \\
$A$ & $\mathrm{~min}^{-1}$ & $0.012 \pm 0.003$ & 58 \\
$\gamma$ & & $1.1 \pm 0.3$ & \\
$k_{\text {out }}$ & day $^{-1}$ & $0.015 \pm 0.001$ & 10 \\
$\tau$ & $\mathrm{h}$ & $1.9 \pm 0.6$ & 31 \\
$\quad$ Derived estimates & & & \\
$\quad t_{1 / 2} k_{\text {out }}$ & day & $46 \pm 7$ & \\
Delay onset tolerance & $\mathrm{h}$ & $7.6 \pm 2$ & \\
$\quad(4 \cdot \tau)$ & & $8 \pm 2$ & \\
$\quad t_{1 / 2} S_{\text {max }, 2}$ & min & & \\
\hline
\end{tabular}

tolerance development was observed and on the basis of the combined analysis of s.c. bolus and s.c. continuous infusion, the optimal number of transit compartments could be determined at 4 . The delay in tolerance development, defined as four transit times, was $7.6 \pm 2 \mathrm{~h}$. After a single s.c. bolus injection of $600 \mu \mathrm{mol} \mathrm{kg}{ }^{-1}$, the theoretical hypothermic effect is reduced by $28 \%$ because of tolerance development, whereas after a s.c. continuous infusion of $40 \mu \mathrm{mol} \mathrm{kg}{ }^{-1} \mathrm{~h}^{-1}$, the maximum possible temperature effect was reduced by $66 \%$. This is graphically illustrated in Fig. 6 .

The single dose data did not yield information on the slow turnover of $Q_{0}\left(k_{\text {out } Q}\right)$ and were therefore fitted with $k_{\text {out } Q}$

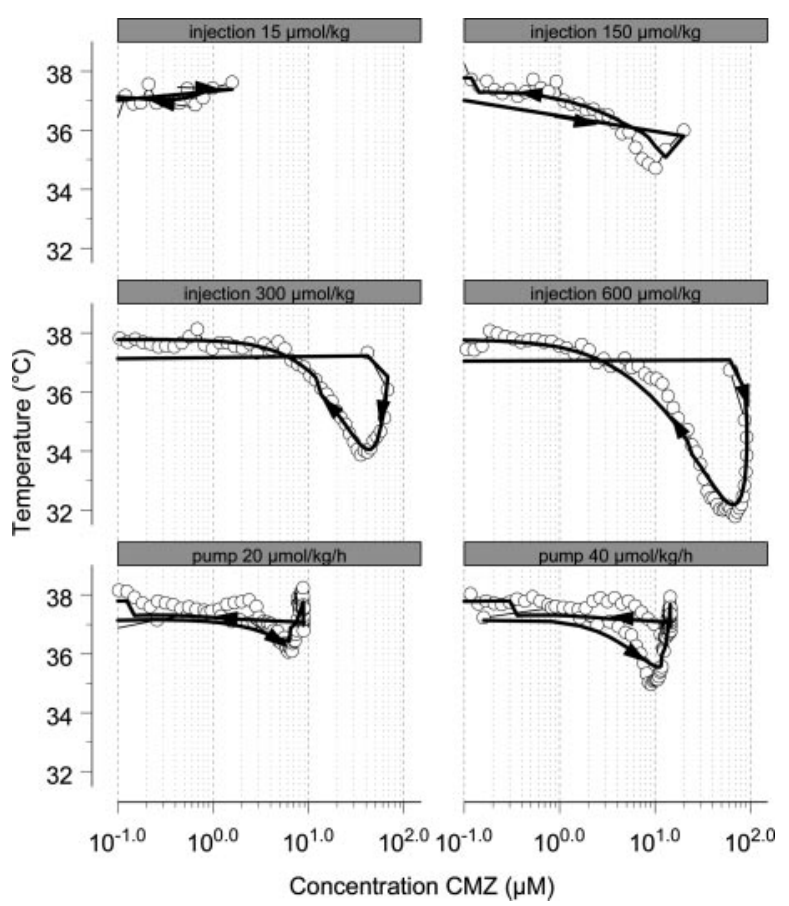

Fig. 5. Individual observed (markers) and fitted individual (solid lines) and population (dotted lines) concentration-effect relationships for typical individuals (same as in Fig. 4) after a single s.c. injection and implantation of an osmotic pump. The loop indicates a delayed effect, and the arrows indicate the time order of the observations. Note that the injections revealed a clockwise loop, whereas the continuous administration revealed a counterclockwise loop.

fixed at $0.022 \mathrm{day}^{-1}$. This corresponded to a turnover time of 32 days. This value was graphically derived from the average minimum effect (mean \pm S.D.) for all individuals measured at 100 min after injection on days 1, 3, 6, 10, 16, 24, and 32 . This relationship is shown in gray in Fig. 7. Thereafter, parameter $k_{\text {out } Q}$ was estimated in the analysis of the repeated dosing data with all other pharmacodynamic parameters fixed at the single dose estimates. The turnover rate constant $k_{\text {out } Q}$ was estimated at $0.015 \pm 0.001(10 \%)$ day $^{-1}$, yielding a turnover time of $46 \pm 3$ days. The results are shown in Fig. 7. The predicted time courses of $Q_{0}$ to $Q_{4}$ are shown in Fig. 8.

The predictive performance of the model was tested by simulation of the profile of the validation data set (Fig. 9). The model successfully predicted the effect on days 1 and 15 but underestimated the effect on day 32 .

\section{Discussion}

In the present investigation, a pharmacodynamic model using various components was put forward for the quantification of the CMZ-induced hypothermia, vehicle treatment, baseline behavior, and tolerance development. Exposure to CMZ was described by means of a pharmacokinetic model with nonlinear elimination. The absorption rate constant was dose-dependent, which is most likely explained by the decrease in $\mathrm{pH}$ of injection fluids with higher doses, resulting in slower absorption. Adjustment of the $\mathrm{pH}$ to physiological values was avoided for reasons of low solubility at physiological $\mathrm{pH}$. The variability observed in the bioavailability may be due to the different occasions of the pharmacokinetic experiments. Nevertheless, the primary goal of the pharma- 

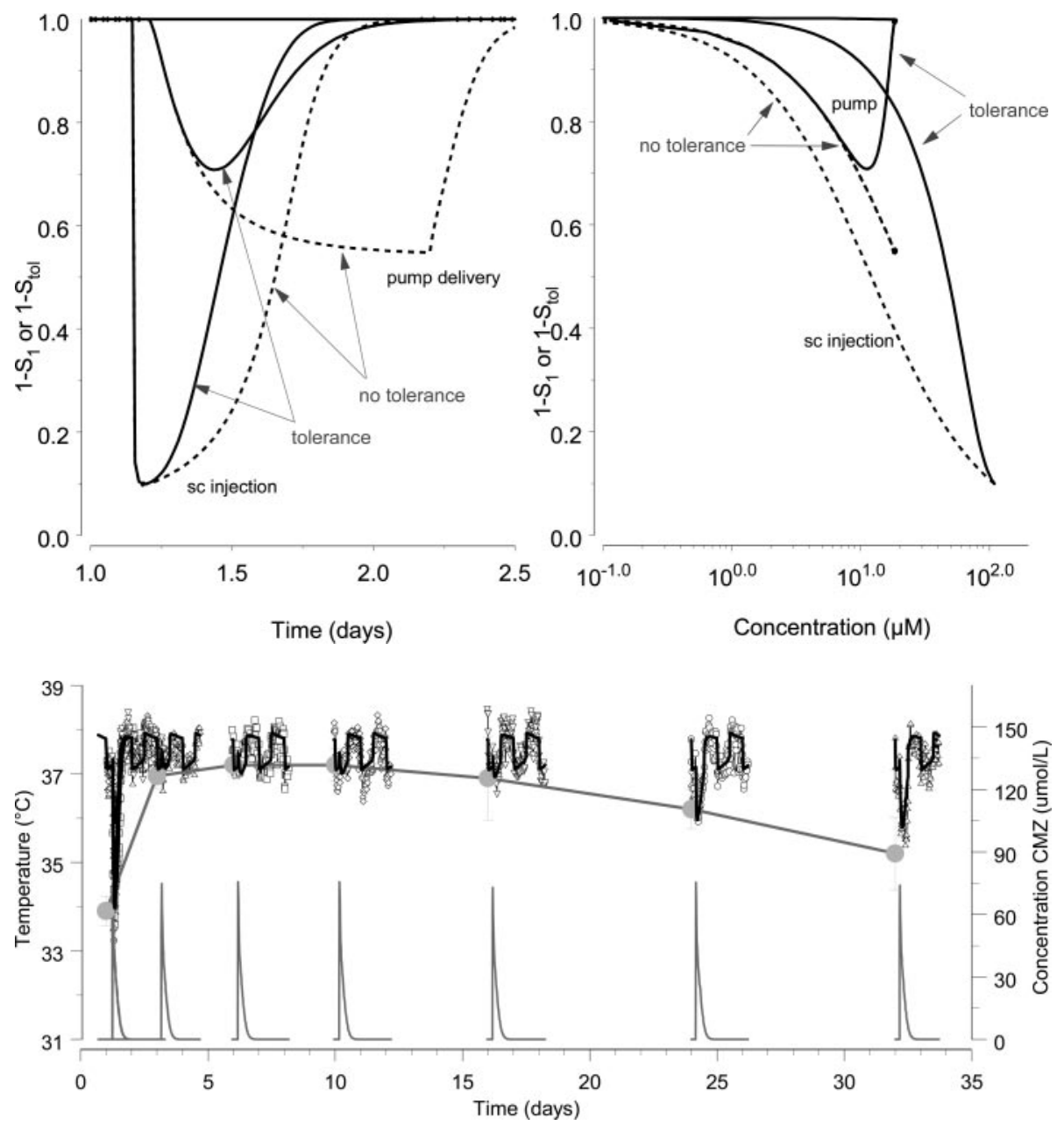

Fig. 6. Left, time course of the CMZ stimulus on temperature with ( $S_{\text {tol }}$, solid line) and without tolerance development $\left(S_{1}\right.$, dotted line). The area under the $S_{\text {tol }}$ curve is $66 \%$ for the injection and $28 \%$ for the pump compared with the $S_{1}$ curve. Right, concentration-stimulus relationship for CMZ on temperature with $\left(S_{\text {tol }}\right.$, solid line) and without tolerance development $\left(S_{1}\right.$, dotted line) cokinetic model was to predict the population exposure profiles as adequately as possible to function as driver of the pharmacodynamics.

To characterize the complex dynamic system with circadian rhythm in baseline, hypothermia, and tolerance development, a systematic approach was adopted to building a pharmacodynamic model. The baseline and vehicle temperature profiles were first analyzed and fixed in the analysis of CMZ-induced temperature decreases. The asymmetric behavior, with a relatively constant temperature during the night followed by a sudden fall of $1^{\circ} \mathrm{C}$ at the start of the day and a gradually increasing temperature during the day, was successfully captured (Achermann and Kunz, 1999; Lobo et al., 1999; Benstaali et al., 2001; Sällström et al., 2005). The advantage of quantifying baseline behavior lies in improving quantification of the pure drug effect for low doses or low efficacy agonists that cannot produce a maximum decrease in temperature. The maximal possible physiological reversible decrease in body temperature is $\sim 5^{\circ} \mathrm{C}$. A larger temperature decrease might result in a very long recovery time or even death. Thus, a circadian rhythm of $1^{\circ} \mathrm{C}$ represents $20 \%$ of the maximum physiological temperature decrease.

The baseline model, combined with an empirical function of temporary elevation of temperature due to handling events, served as model for vehicle treatment. Transient elevation of temperature during insertion of the osmotic pump was lower than after injections, which is probably due to opposing effects of the short period of isoflurane anesthesia necessary for pump implantation and removal (Prudian et al., 1997). However, the significant influence of handling underlines the need to control experimental settings. It may therefore be likely that the size of the drug effect depends on the time of drug administration and experimental conditions such as handling (Lemmer, 1999).

For the temperature regulation, both a turnover model and a feedback model were tested. In the model optimization step, it appeared that a basic turnover model fitted the data reasonably well (Dayneka et al., 1993), although the feedback model performed better due to increased flexibility (Zuideveld et al., 2001). The feedback model might also describe the physiological regulation of the temperature in a more mechanistic way by using a feedback loop. This model, however, might also be preferred in studying a low efficacy agonist as oscillatory behavior of the model (and observations) are more pronounced at low stimulation (Zuideveld et al., 2004). On the other hand, for application in routine analysis, the complexity and calculation time for the feedback model might be a disadvantage (Sällström et al., 2005).

Parameter estimability of the feedback model was improved by rescaling the data to the maximum observed temperature decrease. Combined with a correction for vehicle treatment and baseline (see eq. 23), this enabled the characterization of the pure drug effect. The drug-induced hypothermia and counteracting tolerance could not be indepen- 


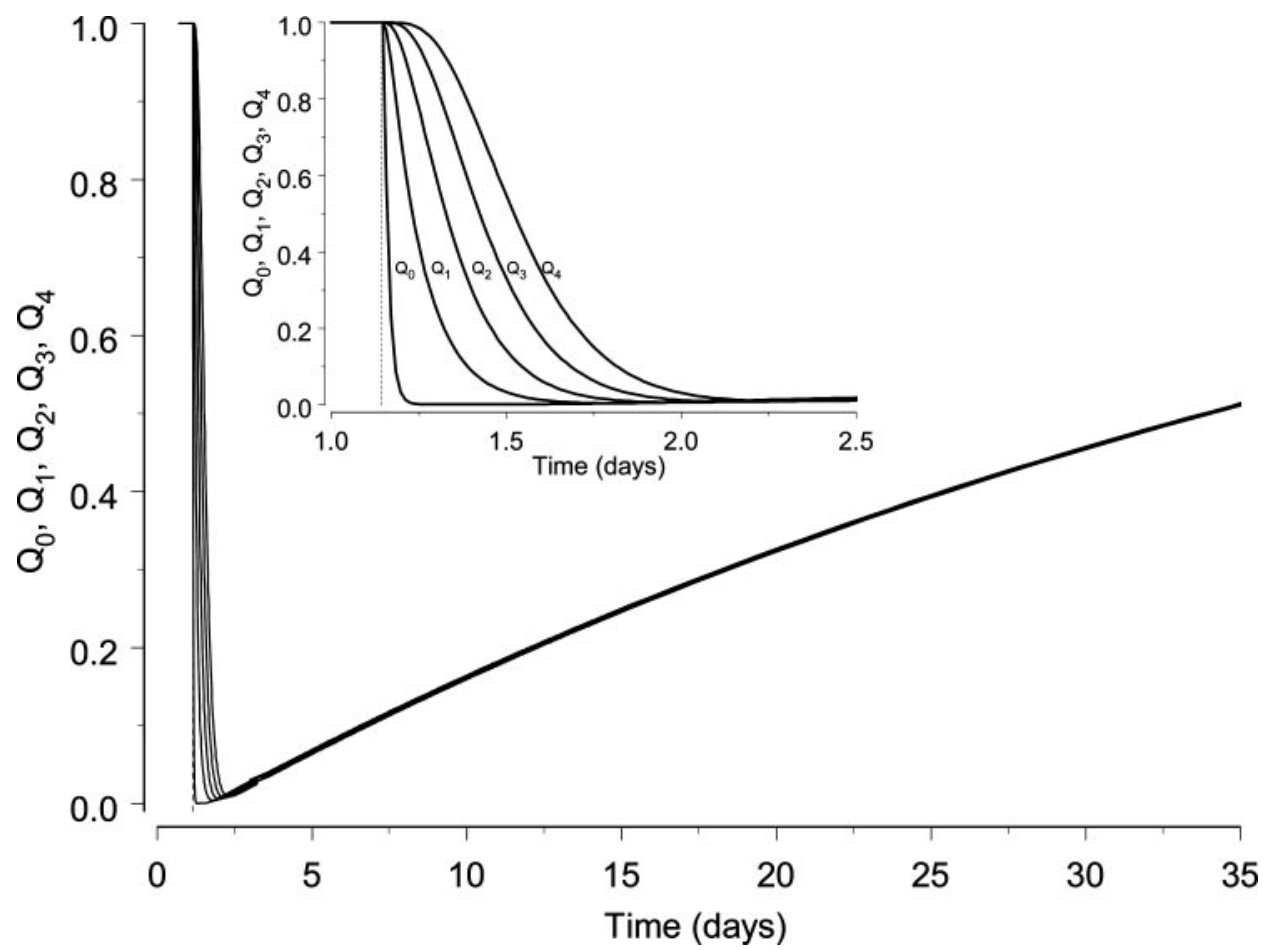

Fig. 8. Time course of the amount in transit compartments $Q_{0}$ to $Q_{4}$. The inset graph shows the first day of the time course in detail. dently measured in a single response profile, but only when multiple doses and infusion rates were analyzed simultaneously. In this investigation, the data obtained by s.c. continuous infusion were crucial for the characterization of the tolerance, whereas the s.c. bolus doses were more informative regarding the temperature regulation (as illustrated in Fig. 6).

The tolerance was modeled by means of a separate turnover model that counteracted the CMZ-induced hypothermic effect and to describe the long-lasting nature of the tolerance. During exposure to CMZ, the compartment $Q_{0}$ was irreversibly emptied with a half-life of $8 \mathrm{~min}$ (see eq. 20). It was estimated via transit compartments that the tolerance development was delayed by $7.6 \pm 2 \mathrm{~h}$. Therefore, the tolerance is less apparent (only $22 \%$ ) after a single

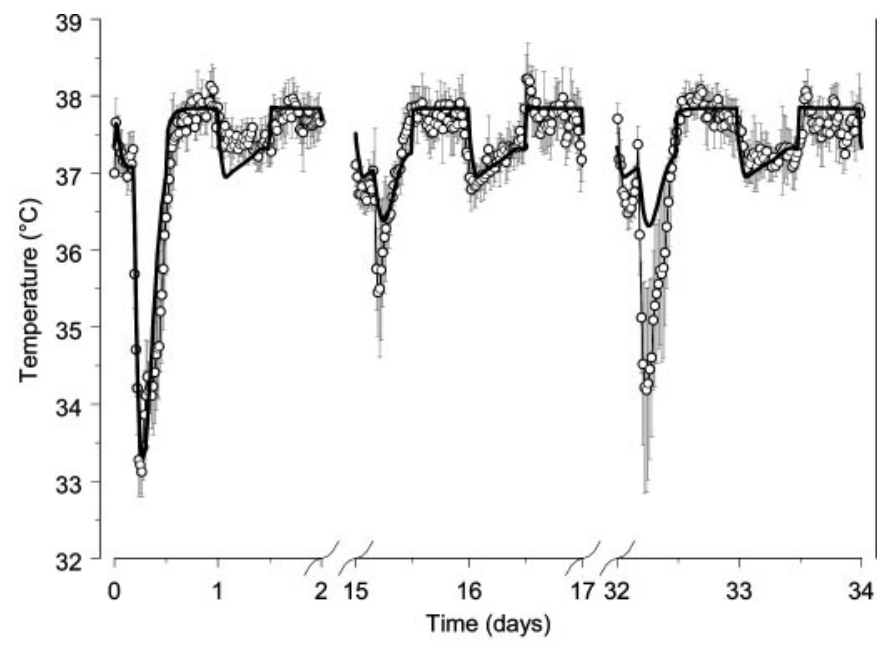

Fig. 9. Prediction of the time course of temperature for the validation data set. Predictions are superimposed on the averaged observations after three occasions of dosing on days 1, 15, and 32. Standard errors of measurements are shown in gray. s.c. bolus injection (a fair amount of CMZ has already been eliminated) but more pronounced (66\%) during s.c. continuous administration. Similar observations are seen with nitroglycerin and nifedipine as examples for which the separation of the beneficial effect and tolerance development to the same effect is highly dependent on the rate of administration (Kleinbloesem et al., 1987; Bauer et al., 1997; Wang et al., 2004). Also a similar tolerance pattern (although less long-lasting) was observed for prolactin release after administration of remoxipride (Movin-Osswald and Hammarlund-Udenaes, 1995).

For tolerance modeling, standard feedback or pool models were not appropriate because of the predicted rebound effects upon withdrawal of the drug. For CMZ, only a slight rebound of $0.3^{\circ} \mathrm{C}$ could be observed during daytime after the highest

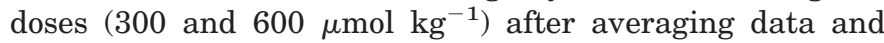
comparing with the baseline. The question is whether this is physiologically significant. It might be the result of a slight disturbance of the circadian rhythm similar to that observed after anesthesia (Prudian et al., 1997). Many approaches to modeling delays between exposure and response have been proposed, such as a link model for distributional delay (Sheiner et al., 1979), a model for slow on/off binding to a receptor (Shimada et al., 1996), various turnover models for timedependent transductions (see Mager and Jusko, 2001 for review), and empirical transit compartments (Sun and Jusko, 1998). Here we have applied transit compartments. The delay in onset of tolerance could indicate that a metabolite of CMZ (Green et al., 2000) is responsible for the observed tolerance instead of CMZ. However, this possibility will not alter the proposed turnover model for tolerance but rather account for a part of the delay as formulated by means of the transit compartments.

The turnover time of $Q_{0}$ was estimated to be $46 \pm 3$ days. This was longer than graphically derived from the data in Fig. 6 as the maximum decrease in temperature does not 
linearly reflect the return of the effect. This long turnover time suggests that to elucidate the mechanism of tolerance, experiments should be designed to find the mediator responsible with a similar turnover rate. It had been shown previously that $\mathrm{GABA}_{\mathrm{A}}$ receptors, 5-hydroxytryptamine ${ }_{1 \mathrm{~A}}$, and $N$-methyl-D-aspartate receptors at least are not involved in tolerance development (Visser et al., 2005). We are not aware of any reports on such an extensive adaptation in a small animal like the rat.

The prediction of the effect after three dose occasions was not completely satisfactory because it underestimated the effect at 32 days, whereas days 1 and 15 were well predicted. Our model assumes that after a second dose of CMZ on day $15, Q_{0}$ is irreversibly emptied again and that the effects of the third dose on day 32 will be almost similar to those of the second dose. However, the remarkable observation was that a third dose gave a larger effect than on day 15 with a larger variability. One reason could be that in this group of rats the actual turnover of $Q_{0}$ was faster and so the predicted effects would be larger. Another factor, which might influence the observations, is that the actual plasma levels during the final dose were higher than simulated. Doses were adjusted to body weight, which was considerable over 32 days but clearance may have remained relatively constant. However, this discrepancy remains to be investigated in further experiments.

The model design was solely dependent on information derived from temperature data. An approach in which mechanistic information is derived from various parts using multiple biomarkers (e.g., the information on the molecular mechanism of the tolerance and circadian rhythm) might improve future generations of complex dynamic models in terms of mechanistic significance. Furthermore, a future step in model development for temperature regulation could be the integration of the model for circadian rhythm into the set-point model in such a way that $T_{\text {ref }}$ is subject to a biorhythm. Another focus could be the independent estimation of $k_{\text {out }}$, with keeping the flexibility of the set-point model. Because of rescaling of the temperature model, it was not possible to independently derive an estimate (see eqs. 9 and 14). However, on the basis of an analysis using an indirect response model, $k_{\text {out }}$ was estimated between 0.006 and $0.02 \mathrm{~min}^{-1}$, yielding a 30- to 80 -min half-life $k_{\text {out }}$. Although the prediction of other dose regimens in routine research would benefit from simpler models with a high predictive value, the development of simpler models should still recognize specific patterns and the overall rate-limiting step in drug response.

For CMZ in particular, it allowed us to estimate the in vivo potency and to predict and understand the absence of hypothermia upon repeated dosing. The modeling of the CMZinduced hypothermia and tolerance provided an example of modeling of various complex dynamic systems such as baseline temperature, handling effects, tolerance development, delays, and temperature regulation.

\section{References}

Achermann P and Kunz H (1999) Modeling circadian rhythm generation in the suprachiasmatic nucleus with locally coupled self-sustained oscillators: phase shifts and phase response curves. J Biol Rhythms 14:460-468.

Ackerman E, Rosevear JW, and McGuckin WF (1964) A mathematical model of the glucose-tolerance test. Phys Med Biol 9:203-213.

Bauer JA, Balthasar JP, and Fung HL (1997) Application of pharmacodynamic modeling for designing time-variant dosing regimens to overcome nitroglycerin tolerance in experimental heart failure. Pharm Res 14:1140-1145.

Benstaali C, Mailloux A, Bogdan A, Auzeby A, and Touitou Y (2001) Circadian rhythms of body temperature and motor activity in rodents their relationships with the light-dark cycle. Life Sci 68:2645-2656.

Blatteis CM (2000) Thermoregulation in complex situations: combined heat exposure, infectious fever and water deprivation. Int $J$ Biometeorol 44:31-43.

Briese E (1998) Normal body temperature of rats: the set point controversy. Neurosci Biobehav Rev 22:427-436.

Chakraborty A, Krzyzanski W, and Jusko WJ (1999) Mathematical modeling of circadian cortisol concentrations using indirect response models: comparison of several methods. J Pharmacokinet Biopharm 27:23-43.

Chaulk D, Wells J, Evans S, Jackson D, and Corbett D (2003) Long-term effects of clomethiazole in a model of global ischemia. Exp Neurol 182:476-482.

Corbett D, Evans S, Thomas C, Wang D, and Jonas RA (1990) MK-801 reduced cerebral ischemic injury by inducing hypothermia. Brain Res 514:300-304.

Cross AJ, Jones JA, Baldwin HA, and Green AR (1991) Neuroprotective activity of chlormethiazole following transient forebrain ischaemia in the gerbil. $\mathrm{Br} J \mathrm{Phar}$ macol 104:406-411.

Dayneka NL, Garg V, and Jusko WJ (1993) Comparison of four basic models of indirect pharmacodynamic responses. J Pharmacokinet Biopharm 21:457-478.

Ekblad EB and Licko V (1984) Invariant relation between total acid secretion and secretagogue exposure: secretory dynamics in bullfrog. Am J Physiol 246:G325 G330.

Fattinger K, Verotta D, and Benowitz NL (1997) Pharmacodynamics of acute tolerance to multiple nicotinic effects in humans. J Pharmacol Exp Ther 281:12381246.

Gabrielsson J and Weiner D (1997) Pharmacokinetic and Pharmacodynamic Data Analysis, Concepts and Applications. Swedish Pharmaceutical Press, Stockholm.

Gardmark M, Brynne L, Hammarlund-Udenaes M, and Karlsson MO (1999) Interchangeability and predictive performance of empirical tolerance models. Clin Pharmacokinet 36:145-167.

Green AR (1998) Clomethiazole (Zendra) in acute ischemic stroke: basic pharmacology and biochemistry and clinical efficacy. Pharmacol Ther 80:123-147.

Green AR, Murray TK, Misra A, Snape MF, Jones JA, and Cross AJ (2000) The metabolism of clomethiazole in gerbils and the neuroprotective and sedative activity of the metabolites. Br J Pharmacol 129:95-100.

Hempel G, Karlsson MO, de Alwis DP, Toublanc N, McNay J, and Schaefer HG (1998) Population pharmacokinetic-pharmacodynamic modeling of moxonidine using 24-hour ambulatory blood pressure measurements. Clin Pharmacol Ther 64: $622-635$.

Holford NH and Sheiner LB (1982) Kinetics of pharmacologic response. Pharmacol Ther 16:143-166.

Kleinbloesem CH, van Brummelen P, and Breimer DD (1987) Nifedipine: relationship between pharmacokinetics and pharmacodynamics. Clin Pharmacokinet 12 $12-29$.

Lemmer B (1999) Chronopharmacokinetics: implications for drug treatment. J Pharm Pharmacol 51:887-890.

Lobo LL, Claustrat B, Debilly G, Paut-Pagano L, Jouvet M, and Valatx JL (1999) Hypoprolactinemic rats under conditions of constant darkness or constant light: effects on the sleep-wake cycle, cerebral temperature and sulfatoxymelatonin levels. Brain Res 835:282-289.

Mager DE and Jusko WJ (2001) Pharmacodynamic modeling of time-dependent transduction systems. Clin Pharmacol Ther 70:210-216.

Mandema JW and Wada DR (1995) Pharmacodynamic model for acute tolerance development to the electroencephalographic effects of alfentanil in the rat. J Pharmacol Exp Ther 275:1185-1194.

Movin-Osswald G and Hammarlund-Udenaes M (1995) Prolactin release after remoxipride by an integrated pharmacokinetic-pharmacodynamic model with intra and interindividual aspects. J Pharmacol Exp Ther 274:921-927.

Perachon S, Betancur C, Pilon C, Rostene W, Schwartz JC, and Sokoloff P (2000) Role of dopamine D3 receptors in thermoregulation: a reappraisal. Neuroreport 11:221-225.

Porchet HC, Benowitz NL, and Sheiner LB (1988) Pharmacodynamic model of tolerance: application to nicotine. J Pharmacol Exp Ther 244:231-236.

Prudian F, Gantenbein M, Pelissier AL, Attolini L, and Bruguerolle B (1997) Daily rhythms of heart rate, temperature and locomotor activity are modified by anaesthetics in rats: a telemetric study. Naunyn-Schmiedeberg's Arch Pharmacol 355: $774-778$.

Sällström B, Visser SAG, Forsberg T, Ericson AC, Peletier LA, and Gabrielsson $\mathrm{J}$ (2005) A new pharmacodynamic turnover model capturing asymmetric circadian baseline of body temperature, heart rate and blood pressure in rats: Challenges in terms of tolerance and animal handling effects. J Pharmacokin Pharmacodyn 32:835-859 DOI: 10.1007/s10928-005-0087-2.

Salmi P and Ahlenius S (1998) Evidence for functional interactions between 5-HT1A and 5-HT2A receptors in rat thermoregulatory mechanisms. Pharmacol Toxicol 82:122-127.

Sheiner LB, Stanski DR, Vozeh S, Miller RD, and Ham J (1979) Simultaneous modeling of pharmacokinetics and pharmacodynamics: application to dtubocurarine. Clin Pharmacol Ther 25:358-371.

Shimada S, Nakajima Y, Yamamoto K, Sawada Y, and Iga T (1996) Comparative pharmaco-dynamics of eight calcium channel blocking agents in Japanese essential hypertensive patients. Biol Pharm Bull 19:430-437.

Simon E, Pierau FK, and Taylor DC (1986) Central and peripheral thermal control of effectors in homeothermic temperature regulation. Physiol Rev 66:235-300.

Sun YN and Jusko WJ (1998) Transit compartments versus gamma distribution function to model signal transduction processes in pharmacodynamics. J Pharm Sci 87:732-737.

Visser SAG, Pozarek S, Martinsson S, Forsberg T, Ross SB, and Gabrielsson J (2005) 
Rapid and long-lasting tolerance to clomethiazole-induced hypothermia in the rat. Eur J Pharmacol 512:139-151.

Wakelkamp M, Alvan G, Gabrielsson J, and Paintaud G (1996) Pharmacodynamic modeling of furosemide tolerance after multiple intravenous administration. Clin Pharmacol Ther 60:75-88.

Wang EQ, Balthasar JP, and Fung HL (2004) Pharmacodynamics of in vivo nitroglycerin tolerance in normal conscious rats: effects of dose and dosing protocol. Pharm Res 21:114-120.

Zarrindast MR and Oveissi Y (1988) $\mathrm{GABA}_{\mathrm{A}}$ and $\mathrm{GABA}_{\mathrm{B}}$ receptor sites involvement in rat thermoregulation. Gen Pharmacol 19:223-226.

Zuideveld KP, Maas HJ, Treijtel N, Hulshof J, van der Graaf PH, Peletier LA, and Danhof M (2001) A set-point model with oscillatory behavior predicts the time course of 8-OH-DPAT-induced hypothermia. Am $J$ Physiol 281:R2059 R2071

Zuideveld KP, van der Graaf PH, Newgreen D, Thurlow R, Petty N, Jordan P, Peletier LA, and Danhof M (2004) Mechanism-based pharmacokineticpharmacodynamic modeling of $5-\mathrm{HT}_{1 \mathrm{~A}}$ receptor agonists: estimation of in vivo affinity and intrinsic efficacy on body temperature in rats. J Pharmacol Exp Ther 308:1012-1020.

Address correspondence to: Dr. Sandra A. G. Visser, PK/PD Section, DMPK\&BAC, AstraZeneca R\&D Södertälje, SE-151 85 Södertälje, Sweden. E-mail: sandra.visser@astrazeneca.com 